

Not i on of an ai $r$ bubbl e caused by thr ee- phase boundar y r eact i on

\begin{tabular}{|l|l|}
\hline $\begin{array}{l}\text { jour nal or } \\
\text { publ i cat i on titl e }\end{array}$ & 211th Neeting of the El ectrochemical Soci ety \\
\hline page range & 1188 \\
\hline year & $2007-05$ \\
\hline URL & ht t p: //hdl . handl e. net /10098/5206 \\
\hline
\end{tabular}




\section{Motion of an air bubble caused by three-phase boundary reaction}

Jingyuan Chen, ${ }^{*}$ Masanori Satoh, Koichi Aoki, Department of Applied Physics, University of Fukui,

3-9-1 Bunkyo, Fukui-shi, 910-8507 Japan

When a sensor electrode is inserted in living tissues, piercing oil|water interfaces, the three-phase boundary should be formed. Three-phase boundary is crossing phase and phase that ought to be infinitesimal line in mathematics and actually line of the molecular length. If the functional material can be deposited on this line by reaction at three-phase boundary, it is easy to give nanoscale wire. The width of the boundary may be changed size by contacting angle between two phases and others. It has the charm of variety that during electrode reaction ${ }^{1-4}$. This anticipation leads us to work on the redox behavior of ferrocene at the three-phase boundary. A technical key of the electrochemical measurements at the three-phase boundary have been performed by use of a hemispherical oil drop mounted on a planar electrode in aqueous solution, as is shown in Fig.1. The oil phase contained neutral electroactive species ferrocene without deliberately adding supporting electrolyte, whereas the aqueous phase contained electrolyte without electroactive species. The steady-state current potential curve was obtained at $E>0.5 \mathrm{~V}$ (Fig.1), because of a double layer only can be formed at the water|electrode interface by the supporting electrolyte. Since the aqueous phase contains no electroactive species, no electrode reaction occurs at the water electrode interface. Electrode reactions can react only at the three-phase boundary.

We report here is not only leads to understanding iontransport, electrode reaction and interfacial energy near the three-phase boundary, but also help to evaluating accurately the thickness of the boundary from the diffusion current.

The electrochemical measurement presented here is set an air bubble on the top of oil droplet, as is shown in Fig.2B. The voltammogram showed the steady-state anodic wave for the oxidation of ferrocene, independent of potential sweep rates. The limiting current was proportional to the radii of the droplet. These facts indicate that the electrode reaction should occur at the thin annular part on the electrode to which the water and the oil phase merge. However, the boundary has micrometer width that was evaluated from the limiting current on the basis of the micro-band model, much larger than a molecular length or thickness of a diffuse double layer predicted geometrically from intersection of two plains. The large values suggest participation in fluctuation of the boundary. The fluctuation is ascribed to variation of the surface tension owing to forced electrode reaction of ferrocene in the boundary. The surface tension can be determined by the force of motion of an air bubble at oil|water interface.

The electrochemical oxidation of $\mathrm{Fc}$ at the boundary gave rise to motion of the bubble at oil $\mid$ water interface. Variation of surface tension was determined by tracing the bubble with a video microscope (Fig.2B). The moving distance, $x$, between the top of the air bubble and the air|oil interface were evaluated from image analysis with computer software. When the spherical air bubble with the radius, $a$, is retained between the oil phase and the water phase. The bubble is supported by the balance between the buoyancy and the surface tension. Letting the distance between the top of the bubble and the $\mathrm{W} \mid \mathrm{O}$ interface be $x$. The surface energies at the $w \mid a$, the o $\mid \mathrm{a}$ and the wlo interface are $\gamma_{\mathrm{w}}, \gamma_{\mathrm{o}}, \gamma_{\mathrm{wo}}$, respectively, the total surface energy $(U)$ is obtain. $U$ is a quadratic function of $x$ The force by $U$ in the $x$-direction is expressed by $f_{s}=$ $\mathrm{d} U / \mathrm{d} x$. The buoyancy is expressed by $f_{\mathrm{b}}$. The equation for the force balance is $f_{s}+f_{b}=0$, as is illustrated in Fig.2A.

As a result, the bubble moved from the top of the hemispherical nitrobenzene droplet to the nitrobenzene phase is owing to the insertion of the ion into the oil phase from $0.2 \mathrm{~V}$ to $0.4 \mathrm{~V}$. The random-motion of bubble is owing to disturbed by convection around the boundary in the potential domain $0.4-0.8 \mathrm{~V}$ vs. $\mathrm{Ag} \mid \mathrm{AgCl}$, causing by oxidation of $\mathrm{Fc}$ in the boundary (Fig.2C). The motion of the bubble was ascribed to not only the interfacial energy but also charge balance, and mass transport.

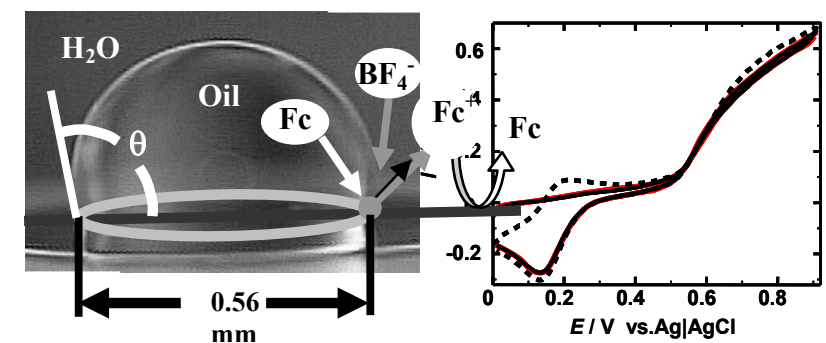

Fig.1. Cyclic voltammogram of the ferrocene-included PDS droplet on the glassy carbon electrode in $0.05 \mathrm{M}$ $\mathrm{NaBF}_{4}$ aqueous solution at $10 \mathrm{mV} \mathrm{s}^{-1}$ for the first (solid curve) and the second (dotted) scan.

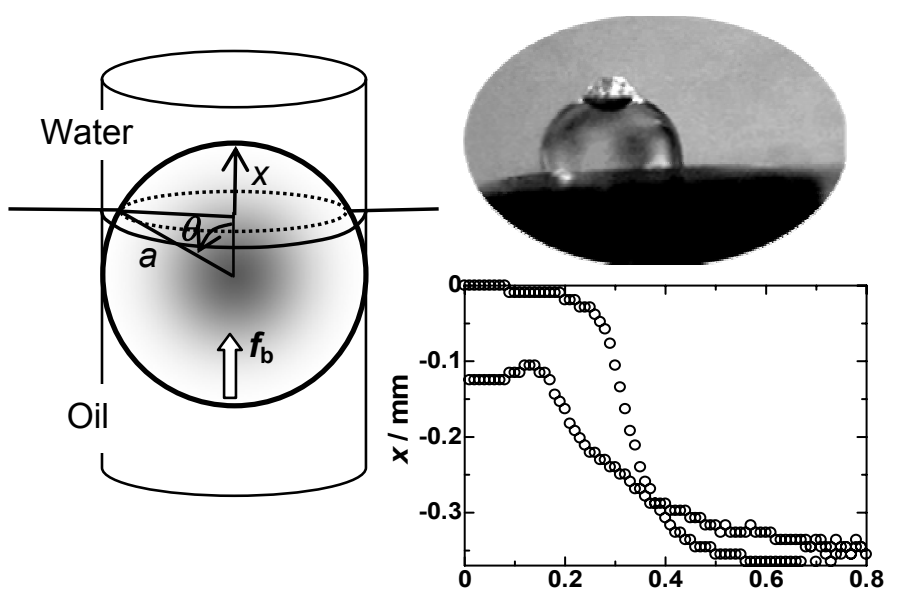

El V vs. $\mathrm{Ag} \mid \mathrm{AgCl}$

Fig.2.

A. Illustration of the spherical air bubble retained between the oil phase and the water phase.

B. The photo of an air bubble on the top of oil droplet on the GC electrode immersed in the aqueous solution. C. Variation of distance, $x$, with applying potential.

[1] P.Tasakorn, J.Chen, K.Aoki, J. Electroanal. Chem., 533 (2002) 119

[2] K.Aoki, P.Tasakorn, J.Chen, J. Electroanal. Chem., 542 (2003) 51.

[3] J.Chen, M.Sato, J. Electroanal. Chem., 572 (2004) 153-159.

[4] K. Aoki, M. Satoh, J. Chen, T. Nishiumi, J.

Electroanal. Chem., 598 (2006) 103-108 